\title{
Brane-world cosmology with black strings
}

\author{
László Á. Gergely \\ Departments of Theoretical and Experimental Physics, University of Szeged, Hungary
}

(Dated: August 9, 2018)

\begin{abstract}
We consider the simplest scenario when black strings / cigars penetrate the cosmological brane. As a result, the brane has a Swiss-cheese structure, with Schwarzschild black holes immersed in a Friedmann-Lemaittre-Robertson-Walker brane. There is no dark radiation in the model, the cosmological regions of the brane are characterized by a cosmological constant $\Lambda$ and flat spatial sections. Regardless of the value of $\Lambda$, these brane-world universes forever expand and forever decelerate. The totality of source terms in the modified Einstein equation sum up to a dust, establishing a formal equivalence with the general relativistic Einstein-Straus model. However in this brane-world scenario with black strings the evolution of the cosmological fluid strongly depends on $\Lambda$. For $\Lambda \leq 0$ it has positive energy density $\rho$ and negative pressure $p$ and at late times it behaves as in the EinsteinStraus model. For (not too high ) positive values of $\Lambda$ the cosmological evolution begins with positive $\rho$ and negative $p$, but this is followed by an epoch with both $\rho$ and $p$ positive. Eventually, $\rho$ becomes negative, while $p$ stays positive. A similar evolution is present for high positive values of $\Lambda$, however in this case the evolution ends in a pressure singularity, accompanied by a regular behaviour of the cosmic acceleration. This is a novel type of singularity appearing in brane-worlds.
\end{abstract}

\section{INTRODUCTION}

There is increasing expectation that general relativity is only the low-energy effective theory of gravitation. This belief originates mainly in the lack of the singularity avoidance of the theory and its still existing failure to be merged with quantum principles. String theory suggests that the five-dimensional Einstein gravity containing our four-dimensional (4D) world as a brane [1] - 3] may be a better approximation. In the original Randall-Sundrum (RS) setup [3], a flat brane was symmetrically embedded into a 5-dimensional anti de Sitter (AdS5) spacetime (the bulk). Later on, generalizations of the model to curved branes and more generic bulk space-times were advanced. In such models, on the brane, gravity is governed by a modified Einstein equation [4]:

$$
G_{a b}=-\Lambda g_{a b}+\kappa^{2} T_{a b}+\widetilde{\kappa}^{4} S_{a b}-\mathcal{E}_{a b},
$$

where $S_{a b}$ denotes a quadratic expression in the energymomentum tensor $T_{a b}$ :

$$
S_{a b}=\frac{1}{12} T T_{a b}-\frac{1}{4} T_{a c} T_{b}^{c}+\frac{1}{24} g_{a b}\left(3 T_{c d} T^{c d}-T^{2}\right),
$$

and $\mathcal{E}_{a b}$ represents the electric part of the Weyl curvature of the bulk. The brane gravitational constant $\kappa^{2}$ and the brane cosmological constant $\Lambda$ are related to the bulk gravitational constant $\widetilde{\kappa}^{2}$, bulk cosmological constant $\widetilde{\Lambda}$ and the (positive) brane tension $\lambda$ through

$$
\begin{aligned}
6 \kappa^{2} & =\widetilde{\kappa}^{4} \lambda, \\
2 \Lambda & =\kappa^{2} \lambda+\widetilde{\kappa}^{2} \widetilde{\Lambda} .
\end{aligned}
$$

In such a scenario our observable universe can be imagined as a Friedmann-Lemaître-Robertson-Walker (FLRW) brane moving in a static Schwarzschild-anti de Sitter (SAdS5) background. Cosmology is modified accordingly, however there is no room left for brane-black holes in this model, which are merely test particles on the brane.

The most simple extension of black holes in higherdimensional gravity is represented by black strings. If black strings populate the bulk, then they penetrate the brane and Schwarzschild black holes emerge. On the brane, black holes could be introduced in the most simple way by a cut and paste method. Replacing spheres removed from the FLRW brane with vacuum regions centered around Schwarzschild black holes we obtain a Swiss-cheese model. In principle such a model could be a more realistic description of the Universe than the FLRW brane, as it contains Schwarzschild black holes with finite mass in the cosmological background. The cosmological consequences can be important, as for example the original general relativistic Swiss-cheese model, the EinsteinStraus model [5] has predicted a modified luminosityredshift relation [6].

It is well known, that in the context of the generalized RS brane-worlds, the generic spherically symmetric black holes are characterized by both a mass $m$ and a tidal charge $q$, the latter originating from the electric part of the bulk Weyl curvature [7]. Thus spherically symmetric brane black holes resemble the Reissner-Nordström solutions, but the tidal charge can take negative values as well, in contrast with general relativity, where $q=Q^{2}$ represents the square of the electric charge $Q$. In spite of the fact that the bulk solution containing such a brane is unknown, various astrophysical phenomena were already studied in this scenario, like gravitational lensing 8]. The tidal charge term represents a correction to the Schwarzschild potential, which scales as $r^{-2}(r$ being the distance measured from the center of symmetry). This correction term comes from the Weyl curvature of the bulk (from the $\mathcal{E}_{a b}$ source term) and it is in fact the most important piece of information we have about the bulk containing the tidal charged brane black hole.

We remark here that the perturbative analysis of 
the gravitational field of a spherically symmetric source in the weak field limit, in the context of the original RS setup has shown other type of corrections to the Schwarzschild potential (which depend on the curvature radius of the fifth dimension) [3], [9]-[10]. These scale as $r^{-3}$, thus they are different from the correction induced by the tidal charge in the tidal charged black hole solution. This shows that the bulk containing the tidal charged black hole brane, under no circumstances can behave as a linearly perturbed AdS5 space-time. This is the second information we have about the bulk containing the tidal charged brane black hole.

The same holds true for the simplest black hole on the brane, given by the Schwarzschild solution (with $q=0$ ). As the Schwarzschild potential does not have any $r^{-3}$ correction term in this case, the bulk containing the Schwarzschild brane black hole is again not a linearly perturbed AdS5 space-time. Remarkably, we do know more on the extension into the bulk of the Schwarzschild brane black hole, than of the generic tidal charged black hole. As conjectured in [1], a Schwarzschild brane black hole can be embedded in the bulk only by extending the singularity into the bulk. Therefore a black string with singular AdS horizon emerges. Recently, the gravity wave perturbations of such a black-string brane-world were computed [12]. It is widely believed, that due to the Gregory-Laflamme instability [13] the black string will shortly decay into a black cigar 14]. However, more recently it was shown that under very mild assumptions, classical event horizons cannot pinch off [15].

The configuration we propose to study in this paper is schematically represented on Fig [ The brane is FLRW and it contains a number of Schwarzschild voids. Irrespective of which region we are on the brane, the electric part of the bulk Weyl curvature is switched off. While this is customary for the cosmological regions (then the bulk becomes AdS5 and there is no dark radiation on the respective brane regions), it does not represent an obvious choice for black strings. As motivation, we note that stable black string solutions with $\mathcal{E}_{a b}=0$ arise in the two brane models, introduced in Ref. [2]. Such black strings extending between the physical brane and a "shadow brane" were already employed as a model of brane-world black holes [12], and we follow the same choice here.

A difficult problem would be to find the global bulk solution for this highly asymmetric configuration. We propose again the cut and paste method here, by inserting a transition zone between the black string / cigar metric and the AdS5 regions. As the exact shape of these regions is not fixed a priori, we conjecture that by choosing an appropriate shape for these regions, the boundary problem becomes solvable. We do not propose to further discuss this topic here, but rather concentrate on the existence of the brane cosmological solution, in the same spirit as brane stellar solutions were discussed in [16- 21], weak gravitational lensing in 81, strong lensing in 22] and galactic rotation curves in [23].

In 24] the Swiss-cheese brane-world model was already

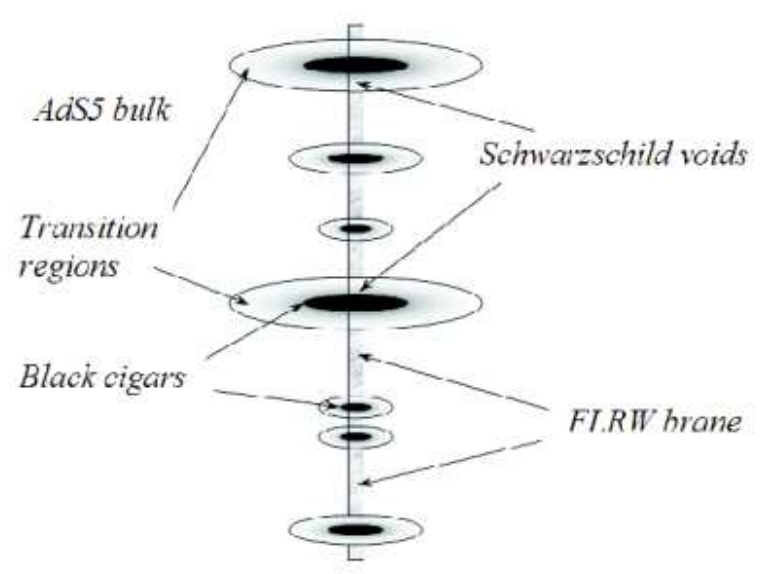

FIG. 1: (color online). A schematic, one-dimensional representation of a Swiss-cheese brane-world. The FLRW brane is embedded in an AdS5 bulk. The brane Schwarzschild black holes extend into the bulk as black strings / cigars. A transition zone separates these black strings / cigars from the AdS5 bulk regions.

studied, assuming $\mathcal{E}_{a b}=0$. By allowing for a cosmological constant $\Lambda$ in the FLRW regions the possibility of having different brane tensions $\lambda$ in the FLRW and Schwarzschild regions of the brane, and / or having different cosmological constants $\widetilde{\Lambda}$ in the bulk regions surrounding them was raised. The junction conditions on the brane have been derived and it has been shown that for static cosmological fluid no such brane (beside the empty one, the Minkowski brane) can exist.

In Section 2 we present the simplest examples of $d y$ namic Swiss-cheese universes on the brane. These consist of black strings / cigars penetrating a cosmological FLRW brane without dark radiation. The intersections appear on the brane as Schwarzschild spheres. We find that contrarily to the static case, the modified Einstein equations admit brane solutions of Swiss-cheese type. We study in detail the cosmological evolution in these models in Section 3. Additional comments on these models, including their classification with respect to the value of the cosmological constant, and remarks on a novel type of pressure singularity emerging in these Swiss-cheese models are presented in the Concluding Remarks.

\section{SWISS-CHEESE BRANE-WORLDS}

We construct the dynamic Swiss-cheese brane- by immersing 4D Schwarzschild vacua with constant comoving radius $\chi_{0}$ in a non-static FLRW brane with spatially flat spatial sections $(k=0)$, cosmological constant $\Lambda$ and tension $\lambda$.

The cosmological evolution in the FLRW regions is governed by the generalized Friedmann and the gener- 
alized Raychaudhuri equation [25]:

$$
\begin{gathered}
\frac{\dot{a}^{2}}{a^{2}}=\frac{\Lambda}{3}+\frac{\kappa^{2} \rho}{3}\left(1+\frac{\rho}{2 \lambda}\right), \\
\frac{\ddot{a}}{a}=\frac{\Lambda}{3}-\frac{\kappa^{2}}{6}\left[\rho\left(1+\frac{2 \rho}{\lambda}\right)+3 p\left(1+\frac{\rho}{\lambda}\right)\right] .
\end{gathered}
$$

General relativity is recovered for $\rho / \lambda \rightarrow 0$. The continuity of the induced metric and of the extrinsic curvature on the junction surfaces between the Schwarzschild vacua and the FLRW regions imply [24]

$$
\begin{aligned}
a \dot{a}^{2} & =\frac{2 m}{\chi_{0}^{3}}, \\
a^{2} \ddot{a} & =-\frac{m}{\chi_{0}^{3}} .
\end{aligned}
$$

Eq. (7) can be easily integrated to obtain the evolution of the scale factor $a$ in cosmological time $\tau$ :

$$
a^{3}=\frac{9 m \tau^{2}}{2 \chi_{0}^{3}} .
$$

Then the other junction condition is satisfied as well. These Swiss-cheese universes therefore forever expand:

$$
\frac{\dot{a}}{a}=\frac{2}{3 \tau}
$$

forever decelerate:

$$
\frac{\ddot{a}}{a}=-\frac{2}{9 \tau^{2}}
$$

and their expansion ceases at $\tau \rightarrow \infty$, as expected for $k=0$. An integration constant was set to zero in Eq. (9) such that the origin of the $\tau$ coordinate is at the Big Bang. From the junction condition (7) and the generalized Friedmann equation (5), the mass of a Schwarzschild void with comoving radius $\chi_{0}$ emerges [24] in terms of the scale factor $a$ and density of the cosmological fluid $\rho$ as:

$$
m=\frac{a^{3} \chi_{0}^{3}}{6}\left[\Lambda+\kappa^{2} \rho\left(1+\frac{\rho}{2 \lambda}\right)\right]
$$

The junction condition (8) and the generalized Raychaudhuri equation (6) give the equation of state of the fluid [24]. Taking into account Eq. (12) this becomes

$$
a^{3}(\rho+p)(\rho+\lambda)=\frac{6 m \lambda}{\kappa^{2} \chi_{0}^{3}} .
$$

Comparison of Eqs. (9) and (12) leads to

$$
\kappa^{2} \rho\left(1+\frac{\rho}{2 \lambda}\right)=-\Lambda+\frac{4}{3 \tau^{2}},
$$

with solutions

$$
\frac{\rho_{1,2}}{\lambda}=-1 \pm \sqrt{1-\frac{2 \Lambda}{\kappa^{2} \lambda}+\frac{8}{3 \kappa^{2} \lambda \tau^{2}}} .
$$

For a positive energy density we choose the + solution and also

$$
3 \Lambda \tau^{2}<4
$$

has to hold. This is immediate for $\Lambda \leq 0$. For any positive $\Lambda$ the energy density is positive for $\tau<\tau_{1} \equiv 2 / \sqrt{3 \Lambda}$ and negative afterwards. For a cosmological constant small enough the positivity of $\rho$ is maintained for a long time even in this case. However, for having $\rho$ real the condition

$$
3\left(2 \Lambda-\kappa^{2} \lambda\right) \tau^{2} \leq 8
$$

should hold, which again is satisfied by any $\Lambda \leq \kappa^{2} \lambda / 2$, but would be clearly violated for $\Lambda>\kappa^{2} \lambda / 2$ at $\tau>$ $\tau_{2} \equiv 2 \sqrt{2 / 3\left(2 \Lambda-\kappa^{2} \lambda\right)}$. We summarize the results for $\rho$ in Table 1

TABLE I: Domains of positivity, negativity and illdefinedness of $\rho$ for various values of $\Lambda$. The constants are $\tau_{1} \equiv 2 / \sqrt{3 \Lambda}$ and $\tau_{2} \equiv 2 \sqrt{2 / 3\left(2 \Lambda-\kappa^{2} \lambda\right)}$.

\begin{tabular}{|c||c|c|c|c|}
\hline$\rho$ & $\tau<\tau_{1}$ & $\tau=\tau_{1}$ & $\tau_{1}<\tau \leq \tau_{2}$ & $\tau>\tau_{2}$ \\
\hline \hline$\Lambda \leq 0$ & + & + & + & + \\
\hline $0<\Lambda \leq \frac{\kappa^{2} \lambda}{2}$ & + & 0 & - & - \\
\hline$\Lambda>\frac{\kappa^{2} \lambda}{2}$ & + & 0 & - & no real solution \\
\hline
\end{tabular}

By inserting Eqs. (9) and (15) in (13) we obtain the evolution of the pressure in cosmological time $\tau$

$$
\frac{p}{\lambda}=1-\frac{4+3\left(\kappa^{2} \lambda-2 \Lambda\right) \tau^{2}}{(3 \lambda)^{1 / 2} \kappa \tau \sqrt{8+3\left(\kappa^{2} \lambda-2 \Lambda\right) \tau^{2}}},
$$

Obviously, neither $p$ is well-defined when $\rho$ is not.

\section{COSMOLOGICAL EVOLUTION}

It is instructive to raise the question, what is the total effective source in the modified Einstein equation (1) for the analyzed Swiss-cheese models. For this, we note that in terms of $\rho, p, a$, the 4-velocity $u^{a}$ and the metric $g_{a b}=$ $-u_{a} u_{b}+a^{2} h_{a b}$, the linear source term is

$$
T_{a b}=\rho u_{a} u_{b}+p a^{2} h_{a b} .
$$

The non-linear source term (2) can be expressed as 25]

$$
\widetilde{\kappa}^{4} S_{a b}=\kappa^{2} \frac{\rho}{\lambda}\left[\frac{\rho}{2} u_{a} u_{b}+\left(\frac{\rho}{2}+p\right) a^{2} h_{a b}\right] .
$$

By employing Eqs. (15) and (18) the total effective source of the modified Einstein equation becomes

$$
-\Lambda g_{a b}+\kappa^{2} T_{a b}+\widetilde{\kappa}^{4} S_{a b}=\frac{4}{3 \tau^{2}} u_{a} u_{b} .
$$




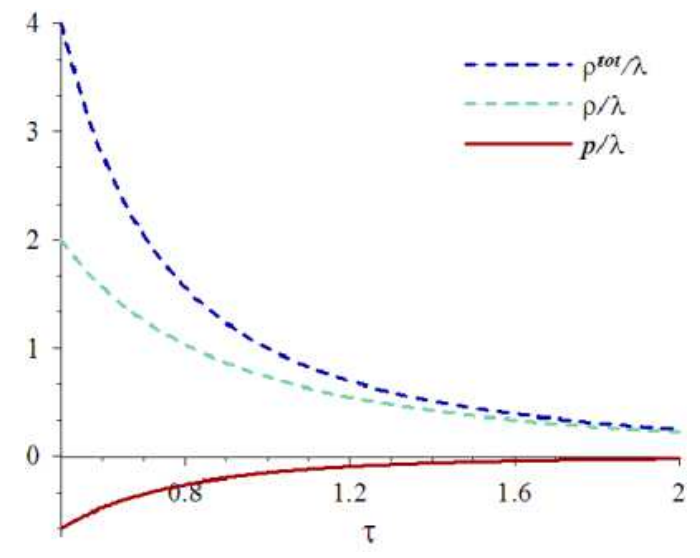

FIG. 2: (color online). Evolution in cosmological time (given in units $\left(4 / 3 \kappa^{2} \lambda\right)^{1 / 2}$ ) of $\rho^{t o t} / \lambda$ (upper curve), $\rho / \lambda$ (middle curve) and of $p / \lambda$ (lower curve), for $\Lambda=0$.

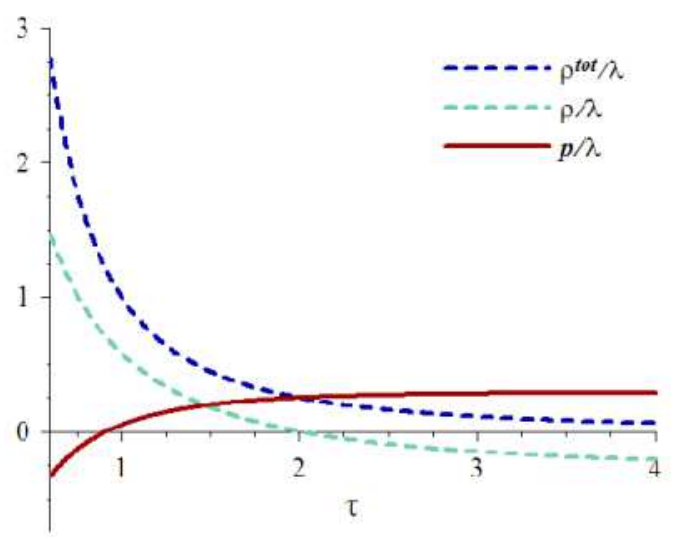

FIG. 3: (color online). As in Fig 2 for $\Lambda=\kappa^{2} \lambda / 4$. We remark that the energy density turns negative at $\tau_{1}$. By contrast, the pressure becomes positive during cosmological evolution. There is a time interval with both $\rho$ and $p$ positive.

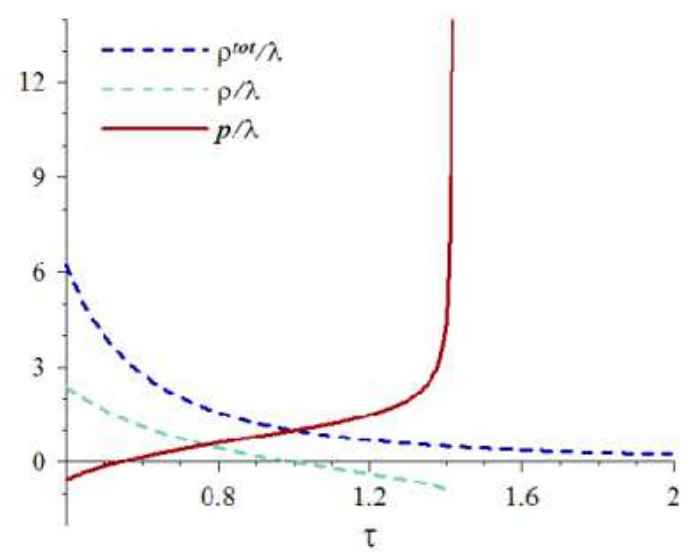

FIG. 4: (color online). As in Fig 2 for $\Lambda=\kappa^{2} \lambda$. The evolution of $\rho$ and $p$ follows the same pattern as in Fig 2 however at $\tau=\tau_{2}$ a pressure singularity appears.
Therefore the FLRW regions effectively behave like filled with a dust with energy density

$$
\rho^{t o t}=\frac{4}{3 \kappa^{2} \tau^{2}}
$$

It is worth to stress that in the general relativistic Einstein-Straus model the fluid is also dust. The comparison of the brane-world and general relativistic Swisscheese models shows the same time dependence of the scale factor, Eq. (9). Also, the time dependence of the dust energy density in the Einstein-Straus model is identical with the time-dependence of the effective total energy density (22) of the brane-world Swiss-cheese model. In the brane-world Swiss-cheese model however the real source is a perfect fluid, with energy density (15) and pressure (18). The brane perfect fluid has three different possible behaviors, depending on the value of the cosmological constant $\Lambda$ in the FLRW regions, as shown in Table I For comparison we illustrate the evolution of both of the energy densities (22) and (15), together with the evolution of the pressure (18) for these three cases. Fig 2 is for $\Lambda=0$, Fig 3 for $\Lambda=\kappa^{2} \lambda / 4$ and Fig 4 for $\Lambda=\kappa^{2} \lambda$. These represent typical behaviors for cosmologies represented in the three lines of Table 【

In all cases the density of the cosmological fluid $\rho$ is less than the total effective density $\rho^{t o t}$. The cosmological evolution (9), identical to that of the Einstein-Straus model, arises as consequence of the modified dynamics of the fluid in the brane-world scenario, given by Eqs. (15) and (18) and represented in Figs 24

How can one interpret the domains of negative energy density occurring whenever $\Lambda>0$ ? In general relativity one can always redefine the energy density and the pressure of an ideal fluid with the aid of a cosmological constant such that the energy conditions are satisfied. Indeed, a perfect fluid characterized by $(\rho, p)$ together with a cosmological constant $\Lambda$ are equivalent to an other perfect fluid with energy density $\bar{\rho}=\rho+\Lambda / \kappa^{2}$ and pressure $\bar{p}=p-\Lambda / \kappa^{2}$ and no cosmological constant. Thus a negative energy density $\rho$ can be turned positive by the above redefinition, provided $\Lambda$ is a sufficiently large positive constant.

Due to the non-linear source terms $S_{a b}$ in the modified Einstein equation (11) the general relativistic redefinition is not in order any more. However in brane-world gravity an other transformation is possible to the new fluid variables $(\bar{\rho}, \bar{p})$ :

$$
\begin{aligned}
& \frac{\bar{\rho}}{\lambda}=-1+\sqrt{1+\frac{2 \Lambda}{\kappa^{2} \lambda}+\frac{2 \rho}{\lambda}\left(1+\frac{\rho}{2 \lambda}\right)}, \\
& \frac{\bar{p}}{\lambda}=1-\frac{1+\frac{2 \Lambda}{\kappa^{2} \lambda}+\frac{\rho}{\lambda}-\frac{p}{\lambda}\left(1+\frac{\rho}{\lambda}\right)}{\sqrt{1+\frac{2 \Lambda}{\kappa^{2} \lambda}+\frac{2 \rho}{\lambda}\left(1+\frac{\rho}{2 \lambda}\right)}},
\end{aligned}
$$

with the result that the cosmological constant is absorbed into the new fluid variables. Then the modified Friedmann and Raychaudhuri equations take the form (5) and 
(6), but with $\Lambda=0$ and $(\bar{\rho}, \bar{p})$ in place of $(\rho, p)$. Therefore the time evolution of the new fluid variables is given by the + branch of Eq. (15) and Eq. (18), both with $\Lambda=0$. Consequently, the new energy density stays positive during the whole evolution, while the new pressure is negative, approaching zero at late times, as shown on Fig. [2]

We conclude that in this brane-world model the evolution of the cosmological fluid is realistic only for $\Lambda \leq 0$. For any positive $\Lambda$ a negative energy density appears, which clearly represents exotic matter. In these cases however the cosmological constant can be transformed out by the redefinition (23) of the fluid variables. The transformation (23) at low energies $(\rho \ll \lambda)$ approaches the general relativistic transformation, while in the high energy / early universe regime $(\rho \gg \lambda)$ it approaches the identical transformation $(\bar{\rho}, \bar{p}) \approx(\rho, p)$, irrespective of the actual value of $\Lambda$.

The question then comes, what is than the invariant meaning of $\Lambda$ ? Originally it was introduced in the model as the cosmological constant in the FLRW regions $\Lambda=$ $\Lambda_{F L R W}$. If we also allow for a cosmological constant $\Lambda_{\text {void }}$ in the voids (so that they become Schwarzschildde Sitter or Schwarzschild anti de Sitter regions instead Schwarzschild black holes), then the junction conditions and the modified gravitational dynamics on the brane give the model presented here, but with $\Lambda=\Lambda_{F L R W}-$ $\Lambda_{\text {void. }}$. Therefore if we allow for the same cosmological constant in both regions on the brane, then $\Lambda=0$ and only the evolution presented on Fig 2 occurs. Otherwise we encounter the pathological cases presented on Figs. 3 and 4

\section{CONCLUDING REMARKS}

We have explicitly constructed Swiss-cheese models by cutting out spheres of constant comoving radius from the FLRW background with flat spatial sections and filling them with Schwarzschild vacua. The latter are the brane sections of black strings (cigars) from the bulk. The junction conditions and cosmological evolution on the brane gave an expanding and decelerating universe. The evolution in cosmological time of the energy density and pressure of the cosmological fluid were given.

For $\Lambda \leq 0$ the energy density is positive and decreasing while the pressure is always negative and increasing. The difference of the behavior of the cosmological fluid in the brane-world Swiss-cheese model as compared to the general relativistic Einstein-Straus model is robust in the early universe. Later on, $\rho \rightarrow \rho^{t o t}$ and $p \rightarrow 0$.

For $0<\Lambda \leq \kappa^{2} \lambda / 2$ the energy density decreases from positive values to negative values (at $\tau>\tau_{1}$ ). By contrast, the pressure increases from negative values to positive ones, switching sign when $\rho=(2 \lambda \Lambda)^{1 / 2} / \kappa$. The fluid evolves differently from the Einstein-Straus model even in the long run. We remark the existence of an epoch, when both the energy density and the pressure of the cosmological fluid on the brane are positive. Even if we exclude the regions with negative energy density from physical grounds, this epoch can represent the static initial state of the static outcome of a dynamic regime of the brane-world evolution .

For $\Lambda>\kappa^{2} \lambda / 2$ the evolution of the cosmological fluid goes through the same sequence of epochs as for $0<\Lambda \leq$ $\kappa^{2} \lambda / 2$, however at $\tau \rightarrow \tau_{2}$ the square root from Eq. (15) tends to zero, thus $\rho \rightarrow-\lambda$ and $p \rightarrow \infty$. This resembles a sudden future singularity [26], however it differs in an important aspect. In spite of the infinite pressure at $\tau=$ $\tau_{2}$, the acceleration given by the Raychaudhuri equation (6) remains finite, as can be seen from Eq. (11). All other derivatives of the scale factor remain regular when this pressure singularity occurs. This is a novel type of singularity arising in brane-wold scenarios and adds to the collection of novel features encountered in the study of brane-world singularities. (For example in Ref. [27] 'quiescent' cosmological singularities were presented, in which the matter density and Hubble parameter remain finite, but all higher derivatives of the scale factor diverge as the cosmological singularity is approached.)

In this paper we have studied the simplest brane-world Swiss-cheese model, consisting of black strings (cigars) penetrating a cosmological FLRW brane without dark radiation. Work in progress on more complicated models, arising by the inclusion an asymmetry across the brane 29] shows that the generic features discussed here are shared by the whole class of brane-world Swiss-cheese models.

Our analysis has shown that in the context of brameworld theories there is not possible to construct a Swisscheese brane-world in which the cosmological fluid is simple (like the dust in general relativity). Indeed, even for the simplest case of $\Lambda=0$ (the same cosmological constant in the FLRW regions and in the Schwarzschild voids), the fluid will obey the condition for dark energy $\rho+3 p<0$ at any $\tau \leq\left(3 \kappa^{2} \lambda\right)^{-1 / 2}$. In spite of the fluid behaving as dark energy, the expansion is decelerated. This is due to the quadratic source term $S_{a b}$, which stays positive and dominates at high energies. Similar features were also encountered in the study of the gravitational collapse on the brane, when the exterior is static [28]. The question comes, whether a more reasonable behavior of the fluid will emerge if we relax the condition $\mathcal{E}_{a b}=0$. This would mean to add tidal charge to the brane black holes and to introduce black holes in the bulk as well. This topic is currently under investigation [30].

\section{ACKNOWLEDGEMENTS}

This work was supported by OTKA grants no. T046939, TS044665, the János Bolyai Scholarship of the Hungarian Academy of Sciences and part of it done during the Pomeranian Workshop in Fundamental Cosmology, Pobierowo, Poland of the COSMOFUN collaboration. I thank the participants for a stimulating atmo- 
sphere and the organizers for support. I also thank Roy
Maartens for comments on brane black holes.
[1] N. Arkani-Hamed, S. Dimopoulos, and G. Dvali, Phys. Lett. B 429, 263 (1998).

N. Arkani-Hamed, S. Dimopoulos, and G. Dvali, Phys. Rev. D 59, 086004 (1999).

[2] L. Randall and R. Sundrum, Phys. Rev. Lett. 83, 3370 (1999).

[3] L. Randall and R. Sundrum, Phys. Rev. Lett. 83, 4690 (1999).

[4] T. Shiromizu, K. Maeda, and M. Sasaki, Phys. Rev. D 62, 024012 (2000).

[5] A. Einstein and E.G. Straus, Rev. Mod. Phys. 17, 120 (1945), errata, ibid. 18, 148 (1946).

[6] R. Kantowski, Astrophys. J. 155, 89 (1969).

[7] N. Dadhich, R. Maartens, P. Papadopoulos, and V. Rezania, Phys. Lett B 487, 1 (2000).

[8] A.S. Majumdar and N. Mukherjee, Int. J. Mod. Phys D 14, 1095 (2005).

[9] J. Garriga and T. Tanaka, Phys. Rev. Lett. 84, 2778 (2000).

[10] S. B. Giddings, E. Katz, and L. Randall, JHEP 0003, 023 (2000).

[11] A. Chamblin, S.W. Hawking, and H.S. Reall, Phys. Rev. D 61, 065007 (2000).

[12] S.S. Seahra, C. Clarkson, and R. Maartens, Phys. Rev. Lett 94, 121302 (2005).

[13] R. Gregory and R. Laflamme, Phys. Rev. Lett. 70, 2837 (1993).

[14] R. Gregory, Classical Quantum Gravity 17, L125 (2000).
[15] G.T. Horowitz and K. Maeda, Phys. Rev. Lett 87, 131301 (2001).

[16] N. Deruelle, Stars on branes: the view from the brane, $g r-q c / 0111065$ (2001).

[17] M. Bruni, C. Germani, and R. Maartens, Phys. Rev. Lett. 87, 231302 (2001).

[18] C. Germani and R. Maartens, Phys. Rev. D 64, 124010 (2001).

[19] R. Casadio, A. Fabbri, and L. Mazzacurati, Phys. Rev. D 65, 084040 (2002).

[20] M. Visser and D.L. Wiltshire, Phys. Rev. D 67104004 (2003).

[21] T. Harko and M.K. Mak, Phys. Rev. D 69064020 (2004).

[22] R. Whisker, Phys. Rev. D 71, 064004 (2005).

[23] M.K. Mak and T. Harko, Phys. Rev. D 70024010 (2004).

[24] L.Á. Gergely, Phys. Rev. D 71, 084017 (2005), erratum ibid. 72, 069902 (2005).

[25] L.Á. Gergely, Phys. Rev. D 68, 124011 (2003).

[26] M. Dabrowski, Phys.Lett. B 625, 184-188 (2005).

[27] Y. Shtanov, V. Sahni, Class.Quant.Grav. 19, L101-L107 (2002).

[28] L.Á. Gergely, Black holes and dark energy from gravitational collapse on the brane, hep-th/0603254 (2006).

[29] L.Á. Gergely and I. Képíró, Asymmetric Swiss-cheese brane-worlds, in preparation (2006).

[30] L.Á. Gergely, Swiss-cheese brane-worlds with tidal charge, in preparation (2006) 